\title{
Application of the Clear Sky Spectral Error for Radiometer Classification in ISO 9060
}

\author{
Stefan Wilbert', Wilko Jessen ${ }^{1}$, Anne Forstinger ${ }^{2}$, Anton Driesse ${ }^{3}$, Aron Habte ${ }^{4}$, \\ Manajit Sengupta $^{4}$, Aitor Marzo ${ }^{5}$, Frank Vignola ${ }^{6}$, Luis F. Zarzalejo ${ }^{7}$ \\ ${ }^{1}$ DLR, German Aerospace Center, Ctra de Senes s/n km4 04200 \\ Tabernas, Spain. Stefan.Wilbert@dlr.de \\ ${ }^{2}$ RWTH Aachen, Templergraben 55, 52062 Aachen, Germany. \\ ${ }^{3}$ Photovoltaic Performance Labs, Emmy-Noether-Str. 2, 79110 Freiburg, Germany \\ ${ }^{4}$ NREL 15013 Denver West Parkway Golden 80401 USA CO \\ ${ }^{5}$ Centro de Desarrollo Energético Antofagasta (CDEA), University of Antofagasta Av. Angamos, \\ 601 Antofagasta 1270300 Chile \\ ${ }^{6}$ Univ. of Oregon, 1252 Univ. of Oregon Eugene OR 97403-1252, USA \\ ${ }^{7}$ CIEMAT, Avda Complutense, 40 Madrid 28040 Spain
}

\begin{abstract}
A recent update of the ISO 9060 standard for the classification of solar radiometers introduces the use of the clear sky spectral error. The spectral error is the change in responsivity that may occur when the spectral distribution of the incident solar radiation differs from the spectral distribution present at the time of calibration. Spectral errors may occur if the radiometer does not have a completely uniform spectral responsivity. This use of the clear sky spectral error is a significant change compared to the previous version of the 9060 standard as now not only thermopile radiometers, but also photodiode radiometers are covered by the standard. We explain the method used to derive the spectral error and present spectral errors for several radiometers of different technologies. The results demonstrate that the new method is helpful to classify radiometers, distinguishing between different radiometer types and excluding inappropriate instruments from the classification as pyranometers and pyrheliometers. We recommend that the method is also used for WMO's radiometer classification which up to our knowledge is currently not fulfilled by any field pyrheliometer and many pyranometers on the market due to its demanding requirements for the spectral responsivity.
\end{abstract}

Keywords: solar spectra, radiometer, spectral error, standardization.

\section{Introduction}

Accurate solar irradiance measurements are essential for the implementation of solar energy power plants and their further development. Radiometer accuracy suffers from different errors, for example related to the incidence angle of the solar radiation or the radiometer temperature. A quite complex measurement error is the spectral irradiance error. It is the error introduced by the change in the spectral distribution of the incident radiation and the deviation of the radiometer's spectral responsivity from a uniform spectral responsivity. While thermopile radiometers have a quite flat spectral responsivity within the wavelength range of interest (here 280 to $4000 \mathrm{~nm}$ ) or at least up to $1800 \mathrm{~nm}$, photodiode sensors have a strongly non-uniform spectral responsivity.

Radiometers are classified according to the ISO 9060 standard (ISO, 2018) in order to improve the comparability of radiation measurements and to make it easier to compare and describe different available instruments. The instrument errors mentioned above are the basis for this classification. The 2018 update of ISO 9060 introduces the clear sky spectral error for a set of clear sky spectra as one of the classification criteria. A given accuracy class can only be reached if the spectral errors of an instrument for all defined test spectra 
relative to the IEC 60904-3 spectra (IEC, 2016) are within the given range for that class. For pyranometers only global horizontal irradiance (GHI) spectra are tested, for pyrheliometers only direct normal irradiance (DNI) spectra.

This new method represents a significant change compared to the previously used classification criterion for spectral characteristics which was called spectral selectivity (ISO, 1990). The spectral selectivity was defined in the ISO standard as the maximum deviation of the spectral responsivity within 350 and $1500 \mathrm{~nm}$ from the mean spectral responsivity within this wavelength interval. The classification with the spectral selectivity excluded photodiode based pyranometers from the standard as these instruments have spectral selectivities of about $300 \%$ - far above the limit for the lowest class (10\%). With the new classification method from (ISO, 2018) selected photodiode radiometers can fulfill the ISO 9060 requirements for the lowest pyranometer class as will be shown in this work.

Another important change in the new version of the ISO 9060 classification from (ISO, 2018) is the possibility to use correction functions to improve the instrument properties relevant to the classification method. Such corrections for temperature or incidence angle effects are commonly used and were not allowed for the classification according to the 1990 version of the standard. The potential for simple correction functions for the spectral error is also analyzed in this paper.

Section 2 of this paper presents the method to derive the clear sky spectral error used for the ISO 9060 classification from (ISO, 2018) including the solar spectra involved in this method. After explaining the calculation of the clear sky spectral error, spectral errors for typical instruments are shown in section 3. Section 4 of the paper discusses the robustness of the method to errors or deviations in the applied spectral responsivity data. Section 5 presents the effect of a simple correction function for the spectral errors on the classification. Finally, section 6 summarizes the work and discusses the results.

\section{Test spectra and calculation of the clear sky spectral error}

The test spectra for which the clear sky spectral error is calculated are clear sky GHI and DNI spectra for nine selected atmospheric conditions and air masses (AM) 1.5 and 5 (not pressure corrected). The nine different atmospheric conditions are summarized in Tab. 1. The software SMARTS 2.9.5 (Gueymard, 2001, Gueymard, 1995) was used to generate the spectra. The spectra for AM 1.5 and the underlying atmospheric conditions are described in detail in (Jessen et al., 2018; Wilbert et al., 2017). The AM of 5 corresponds to a zenith angle of $78.7^{\circ}$ which is close to the $80^{\circ}$ limit used for the definition of the directional error in the 1990 version of ISO 9060. Between $90 \%$ and $99 \%$ of DNI and GHI are received at air masses below 5 depending on the site. AM 1.5 was selected as it is considered to be a good choice for the creation of average spectra (Wilbert et al., 2017). The spectra are available under http://standards.iso.org/iso/9060/ed-2.

Tab. 1: Conditions for the test and reference spectra. $\tau_{500}$ is the aerosol optical depth at $500 \mathrm{~nm}$, PW the precipitable water vapor. The abbreviations for different atmospheres correspond to those from the SMARTS 2.9.5 manual.

\begin{tabular}{cccccc}
\hline Short name & Description & Elev. (km) & $\boldsymbol{\tau}_{\mathbf{5 0 0}}$ & PW (cm) & Atmosphere \\
\hline IEC 60904 & Reference (IEC 60904-3) & 0 & 0.084 & 1.416 & USSA \\
SemClmedHum & Semi-clean, med- humidity, sea level & 0 & 0.27 & 1.416 & USSA \\
SemClHum & Semi-clean, humid, sea level & 0 & 0.27 & 4.115 & TRL \\
HazMedHum & Hazy, medium humidity, sea level & 0 & 0.54 & 1.416 & USSA \\
DustyMedHum & Dusty, medium humidity, sea level & 0 & 0.54 & 1.416 & STS \\
HazHum & Hazy, humid, sea level & 0 & 0.54 & 4.115 & TRL \\
ClDryHi & Clean, dry, high elevation & 1.5 & 0.084 & 0.708 & MLW \\
SemClDryHi & Semi-clean, dry, high elevation & 1.5 & 0.27 & 0.708 & MLW \\
HazDryHi & Hazy, dry, high elevation & 1.5 & 0.54 & 0.708 & STW \\
\hline
\end{tabular}

The spectra deviate strongly from each other both in terms of broadband irradiance and shape. Figures 1 and 2 show the spectra normalized to the integrated broadband irradiance of $900 \mathrm{~W} / \mathrm{m}^{2}$ for DNI and $1000 \mathrm{~W} / \mathrm{m}^{2}$ for GHI. Even after the normalization strong deviations are visible especially for DNI. The normalized spectra are shown because only their shape is relevant for the evaluation of the spectral error as will become clear in the following. The deviations increase with the air mass. 
For GHI the deviations are less pronounced than for DNI. This can be explained by the fact that the aerosol scattering affects DNI much more than it affects GHI. A portion of the DNI scattered by aerosols is still measured by the global pyranometer as DHI irradiance thus making GHI measurements less susceptible to aerosol scattering. A great part of the deviations between the different DNI spectra stems from the aerosol scattering.
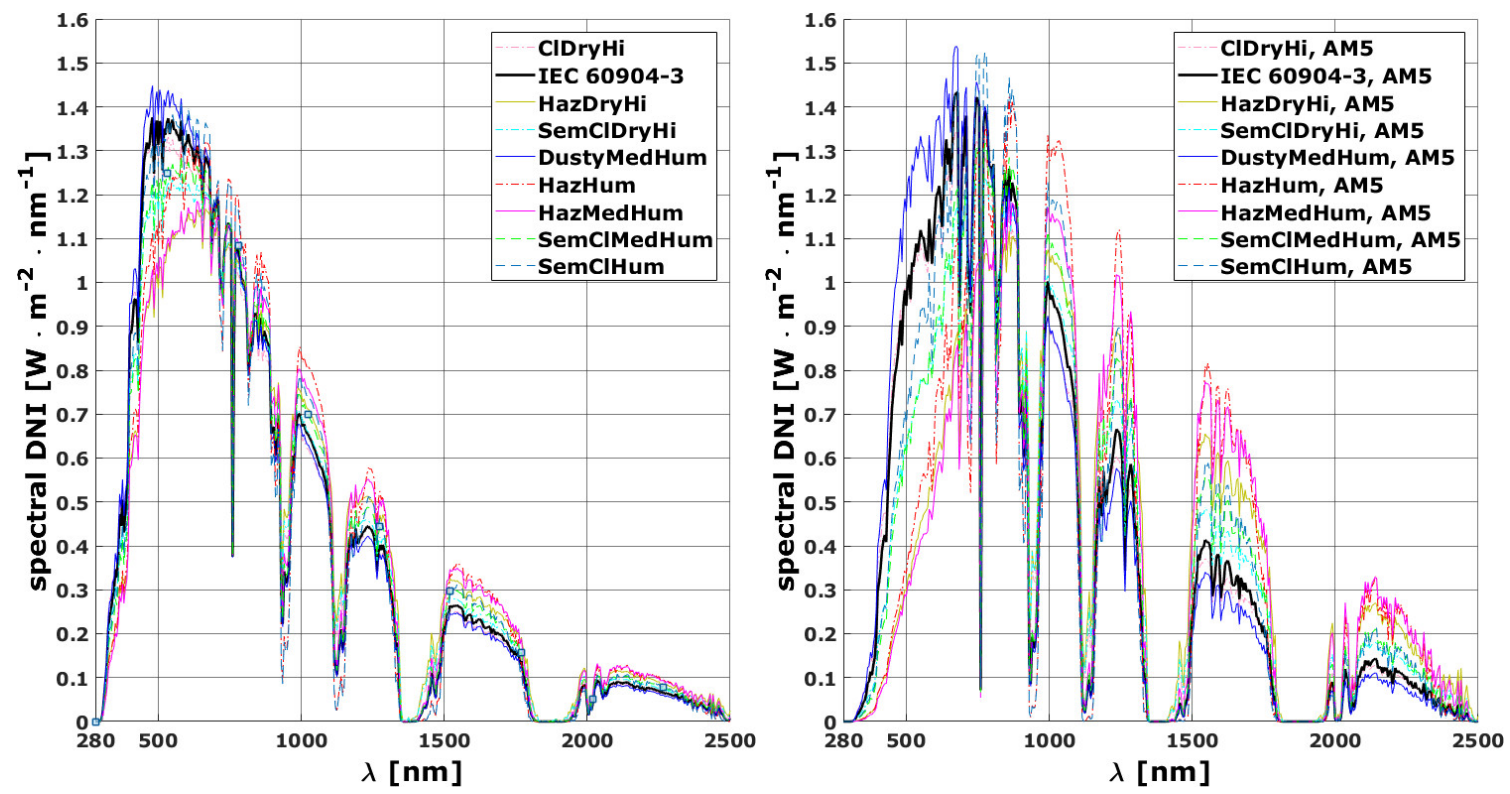

Fig. 1: DNI spectra normalized to the integrated DNI of the IEC 60904-3 spectrum. Left: air mass 1.5. Right: air mass 5.
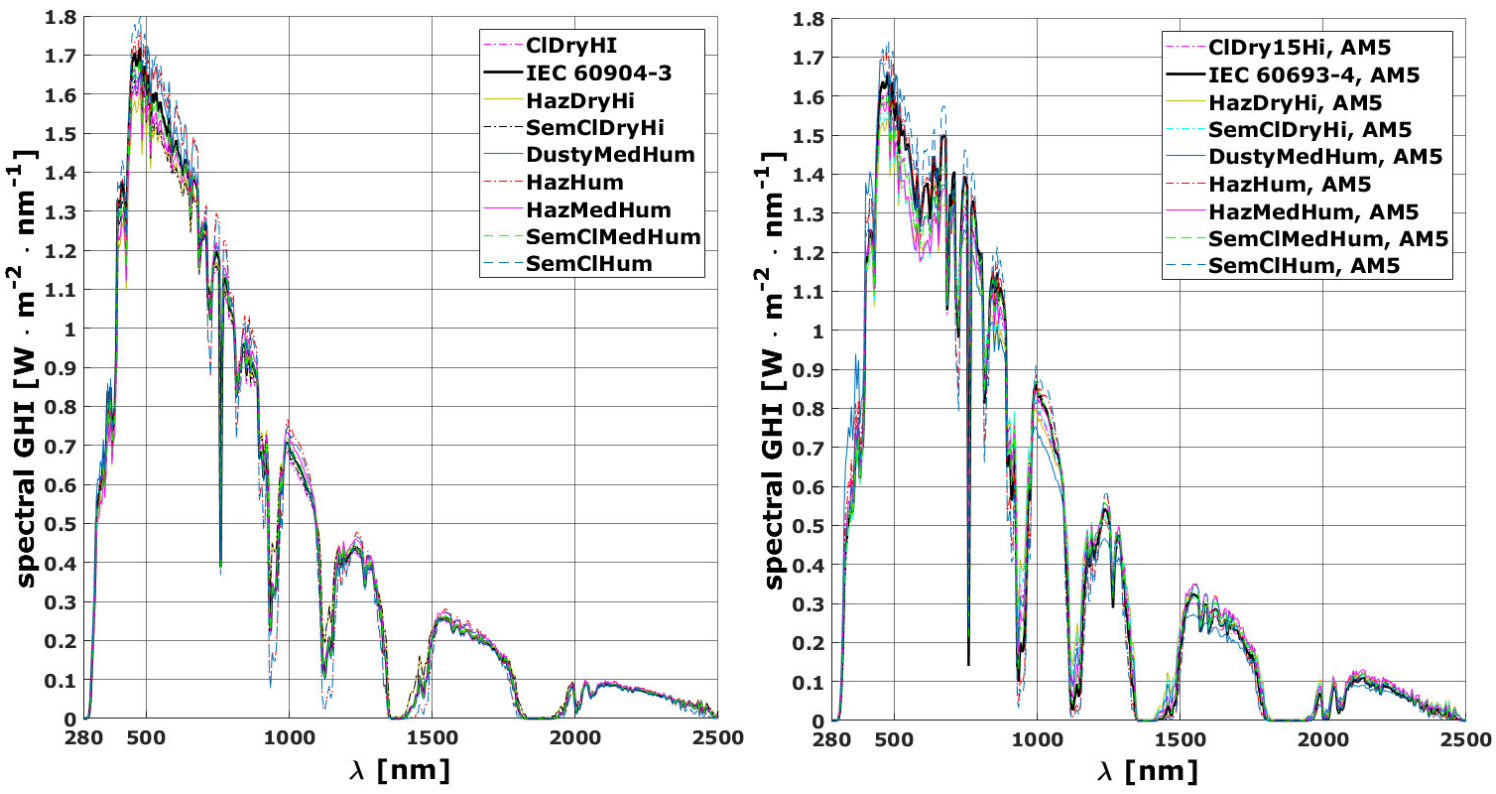

Fig. 2: GHI spectra normalized to the integrated GHI of the IEC 60904-3 spectrum. Left: air mass 1.5. Right: air mass 5.

The spectral errors for a radiometer and the 17 test spectra are calculated relative to a reference spectrum. The reference spectrum $E_{\lambda, \text { Ref }}$ is derived for IEC 60904-3 atmospheric conditions and AM1.5 using SMARTS 2.9.5. GHI spectra are used for pyranometers, DNI spectra for pyrheliometers. The spectral error $\delta_{\mathrm{RS}}$ for a specific radiometer with spectral responsivity $R_{\lambda}$ and the tested spectrum $E_{\lambda, T e s t}$ is calculated as:

$$
\delta_{\mathrm{RS}}=\frac{\sum_{j=1}^{N} R_{\lambda}\left(\lambda_{j}\right) \cdot E_{\lambda, \mathrm{Test}}\left(\lambda_{j}\right) \cdot \Delta \lambda_{j}}{\sum_{j=1}^{N} R_{\lambda}\left(\lambda_{j}\right) \cdot E_{\lambda, \mathrm{Ref}}\left(\lambda_{j}\right) \cdot \Delta \lambda_{j}} \cdot \frac{\sum_{j=1}^{N} E_{\lambda, \mathrm{Ref}}\left(\lambda_{j}\right) \cdot \Delta \lambda_{j}}{\sum_{j=1}^{N} E_{\lambda, \text { Test }}\left(\lambda_{j}\right) \cdot \Delta \lambda_{j}}-1
$$


$\lambda_{j}$ is the $j$-th wavelength of $N$ (from 280 to $4000 \mathrm{~nm}$ ). The step size $\Delta \lambda_{j}$ is between $0.5 \mathrm{~nm}$ and $5 \mathrm{~nm}$. The minuend is the spectral mismatch factor.

The maximum of the spectral errors of a pyrheliometer for all 17 DNI test spectra is called "clear sky spectral DNI error". The maximum for the 17 GHI test spectra and a pyranometer is called "clear sky spectral GHI error". The standard establishes limits of $0.5 \%, 1 \%$ and $5 \%$ for the three pyranometer classes A, B and C and $0.01 \%, 0.2 \%, 1 \%$ and $2 \%$ for the pyrheliometer classes AA, A, B and C. A radiometer reaches a given accuracy class if the clear sky spectral error is within the class' percentage range specified by the standard and if all other criteria for this class related to the other instrument errors are met. It is important to note that the spectral error for different atmospheric conditions is different from this "clear sky spectral irradiance error". Also, the spectral error for DHI measurements is different from that for GHI as discussed in Wilbert et al., 2016. The same holds for reflected or tilted irradiance. According to the standard (ISO, 2018), experimental methods to derive the spectral error for the specified test spectra could be used instead of the above calculation method according to the standard.

\section{Demonstration of the applicability of the classification method}

To demonstrate the classification method and its applicability tests were performed with spectral responsivities of common thermopile pyranometers (CMP11), thermopile pyrheliometers (CHP1) and photovoltaic devices (LI200R pyranometer, LI190R photosynthetically active radiation (PAR) sensor, mono-Si reference cell). The spectral responsivities are shown in Fig. 2. The information for the pyranometers and the PAR sensor was taken from the manufacturer specifications (Kipp\&Zonen, 2006; LICOR, 2005; LI-COR, 2015). The mono-Si cell's responsivity is obtained from (Winter et al. 2009). The spectral response for the pyrheliometer is derived as the product of the spectral transmittance data from the manufacturer specification (Kipp\&Zonen, 2009) and a spectral absorptance measurement of the coated thermopile provided by Mr. van Wely from Kipp\&Zonen. As the spectral transmittance in (Kipp\&Zonen, 2009) is normalized to the maximum of 1 the data were multiplied with the maximum absolute spectral transmittance of a $2 \mathrm{~mm}$ thick Infrasil 301 glass from (Heraeus, 2019) to obtain the absolute spectral transmittance. According to (Kipp\&Zonen, 2008) the pyrheliometer windows are $2 \mathrm{~mm}$ thick and Infrasil 301. The maximum spectral transmittance of such a glass sample is virtually identical to the transmittance caused only by the reflectance on the sample's two surfaces which is also given in (Heraeus, 2019).

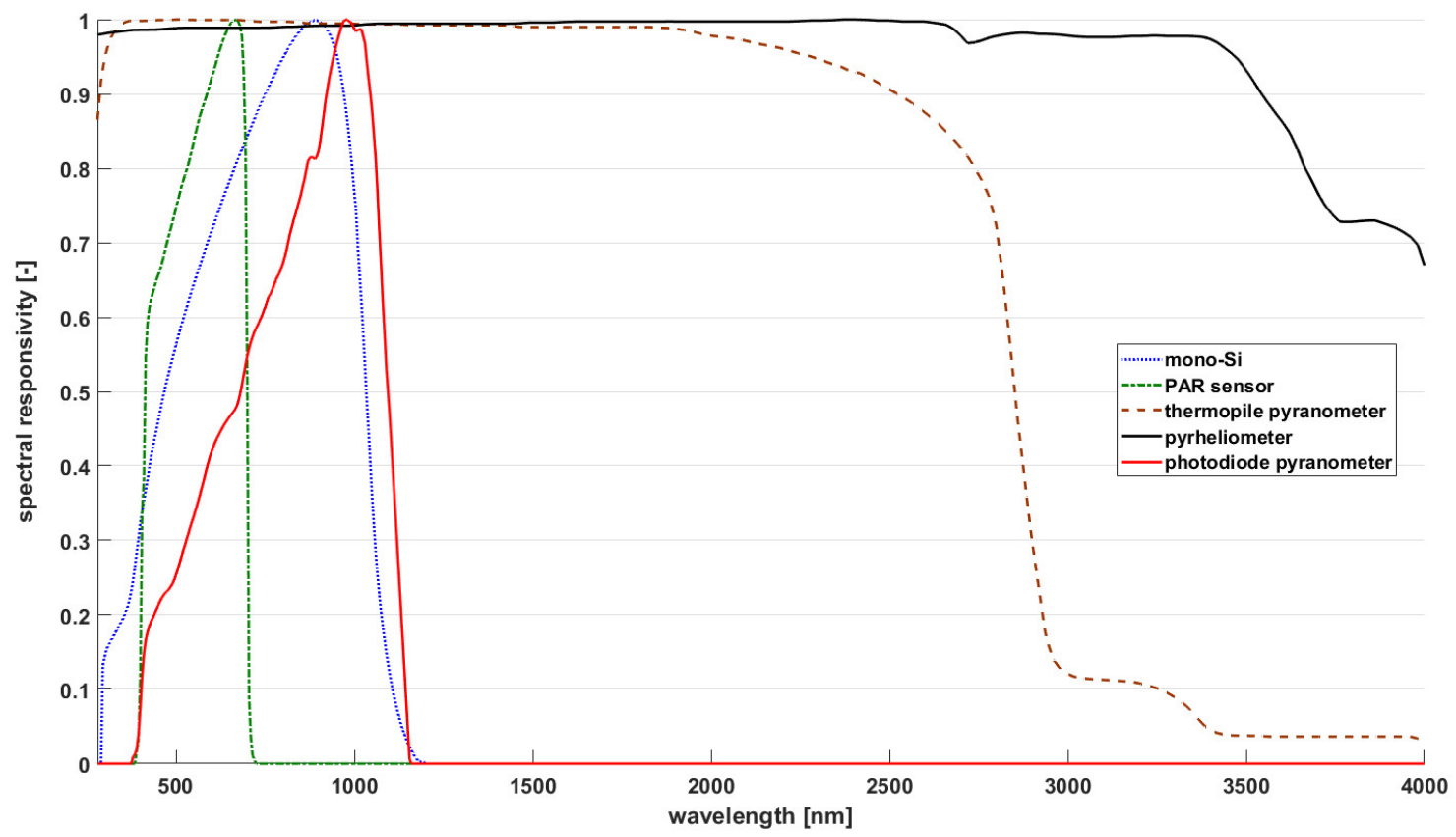

Fig. 3: Spectral responsivities of the tested devices. 
The spectral GHI errors for the exemplary devices are shown in Fig. 4. We used eq. 1 without further corrections for these calculations. The great difference between the thermopile pyranometer and the photovoltaic devices, and the difference between the LI200R and the PAR sensor demonstrate that useful limits for the spectral error can be found for classification. The maximum of the absolute amount of the spectral errors are obtained from the displayed data and shown in Tab. 2. With the limit for the clear sky GHI spectral error of $5 \%$ for the lowest pyranometer class, Si-pyranometers can obtain an ISO 9060:2018 classification if they also fulfill the other criteria related to directional errors, etc.. PAR sensors on the other hand are excluded. Reference cells are not rejected by the spectral error, but most likely by other limits given by the classification scheme, in particular by the directional error. The clear sky spectral errors found for the thermopile pyranometers are below $0.4 \%$ as visible in the zoomed in representation of the previous graph (Fig. 5). A pyranometer with the spectral responsivity of the exemplary pyrheliometer would lead to even lower errors $(0.03 \%)$. This highlights the advantage due to their flat spectral responsivity compared to photovoltaic sensors.

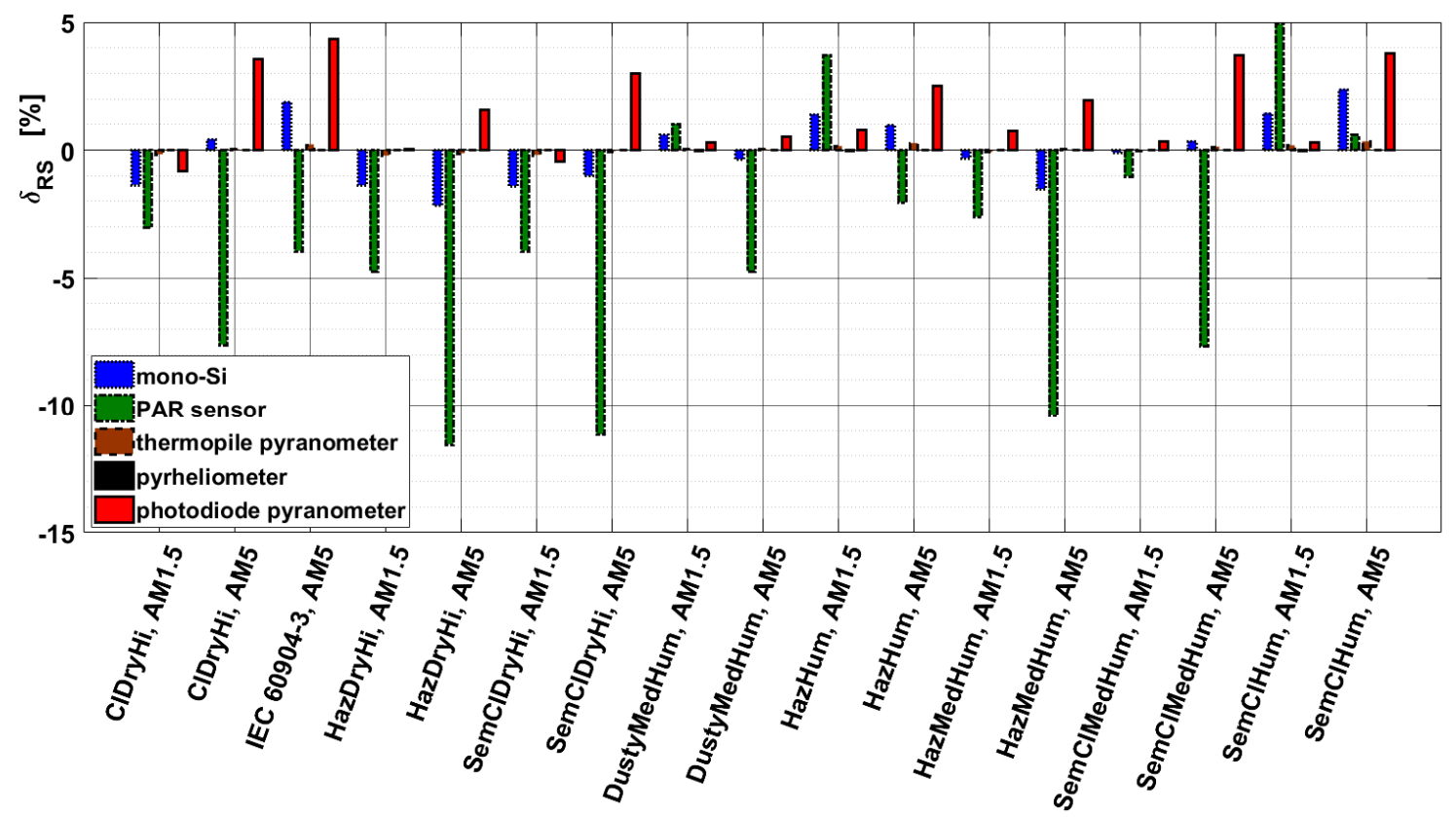

Fig. 4: Spectral errors for exemplary spectral responsivities of radiometers and GHI.

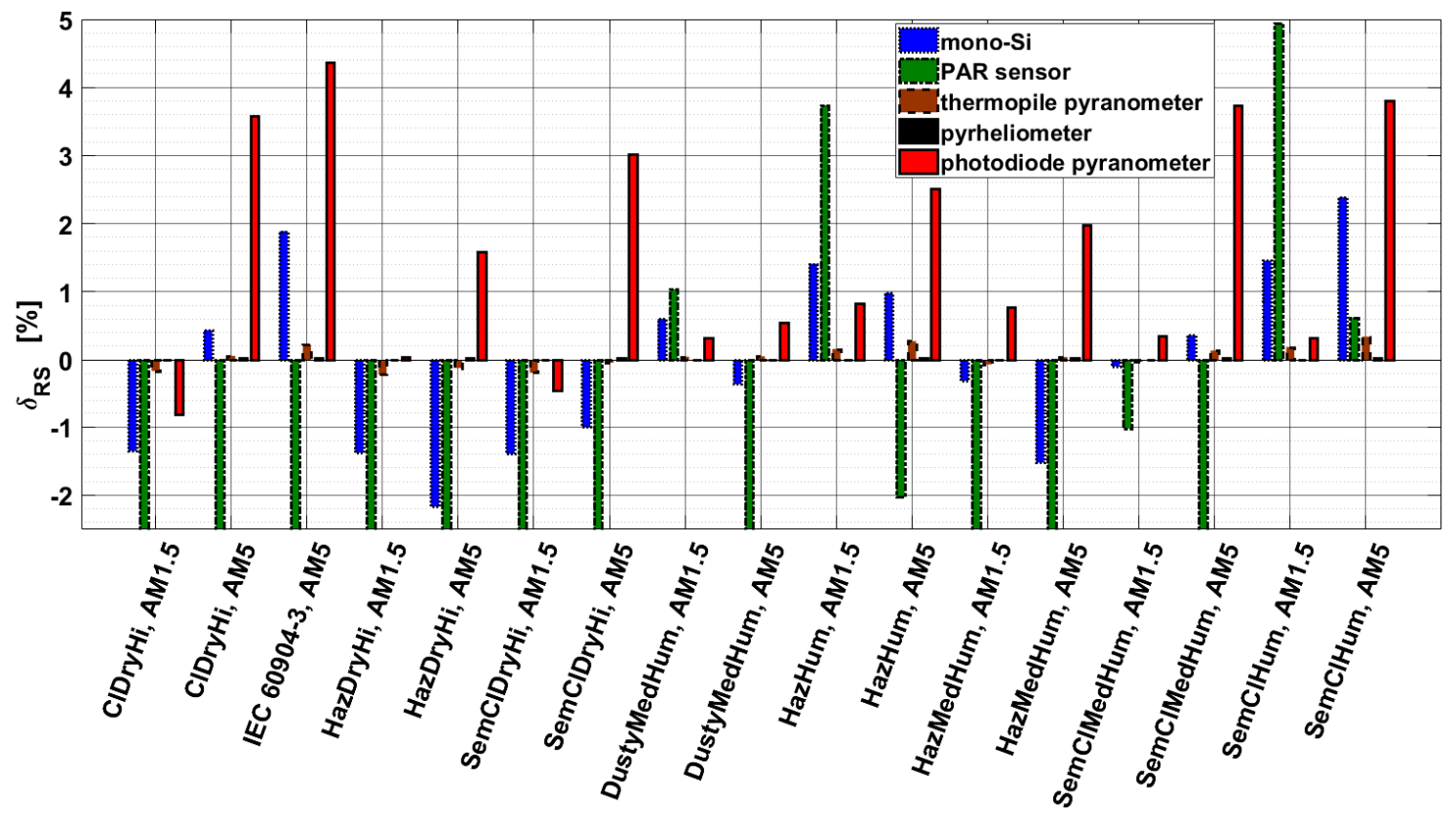

Fig. 5: Spectral errors for exemplary responsivities of radiometers and GHI. Same as Fig. 4 zoomed in. 
Tab. 2: Clear sky spectral error for several spectral responsivities corresponding to different radiometers and a reference cell.

\begin{tabular}{|c|c|c|}
\hline Irradiance component & Spectral responsivity & $\begin{array}{c}\text { Clear sky spectral } \\
\text { irradiance error [\%] }\end{array}$ \\
\hline \multirow{4}{*}{ GHI } & mono-Si & 2.4 \\
\cline { 2 - 3 } & PAR sensor & 11.5 \\
\cline { 2 - 3 } & Thermopile pyranometer & 0.3 \\
\cline { 2 - 3 } & Photodiode pyranometer & 4.4 \\
\cline { 2 - 3 } & Pyrheliometer & 0.03 \\
\hline \multirow{4}{*}{ DNI } & mono-Si & 26.2 \\
\cline { 2 - 3 } & PAR sensor & 63.8 \\
\cline { 2 - 3 } & Thermopile pyranometer & 1.7 \\
\cline { 2 - 3 } & Photodiode pyranometer & 10.0 \\
\cline { 2 - 3 } & Pyrheliometer & 0.1 \\
\hline
\end{tabular}

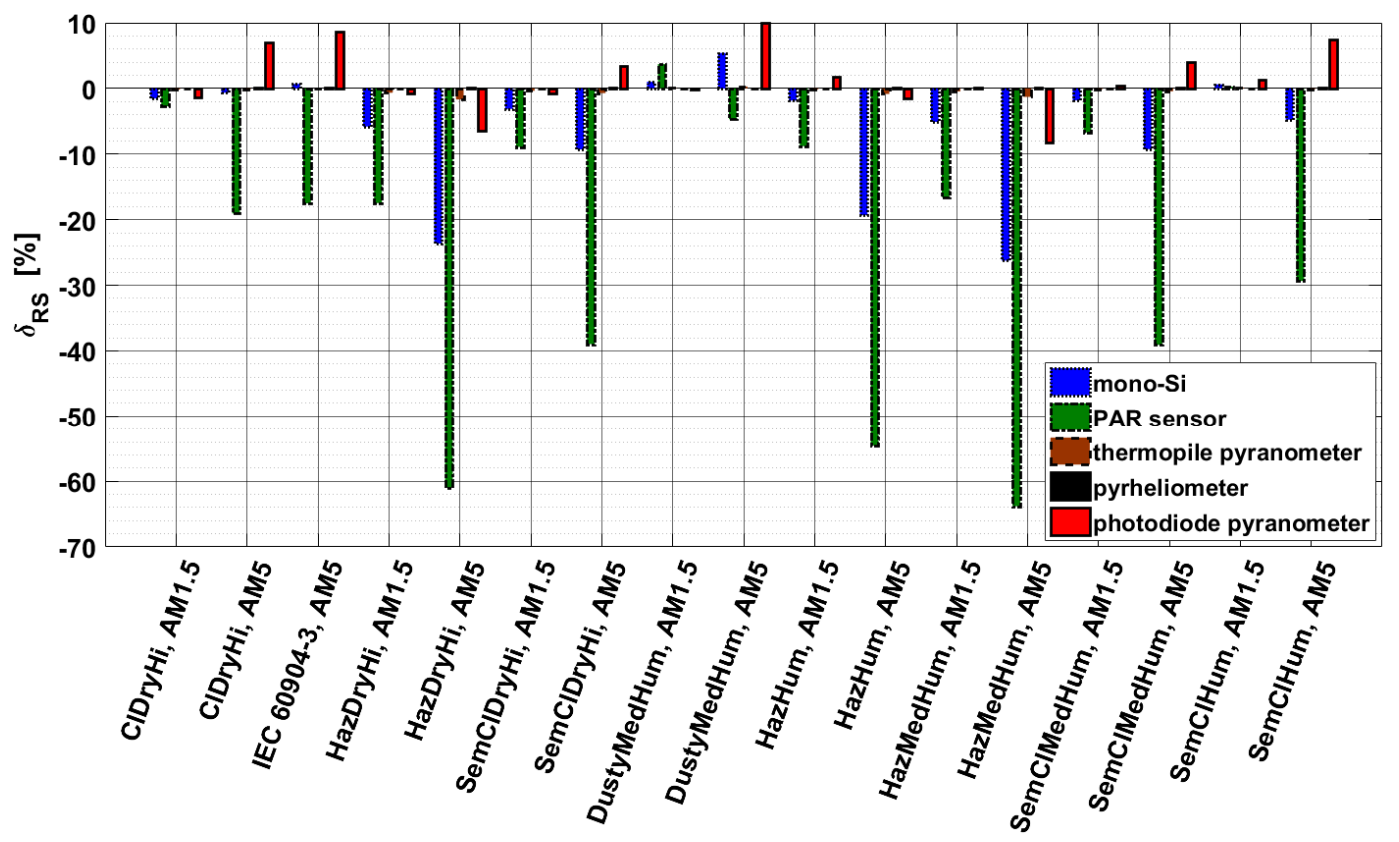

Fig. 6: Spectral errors for exemplary responsivities of radiometers and DNI.

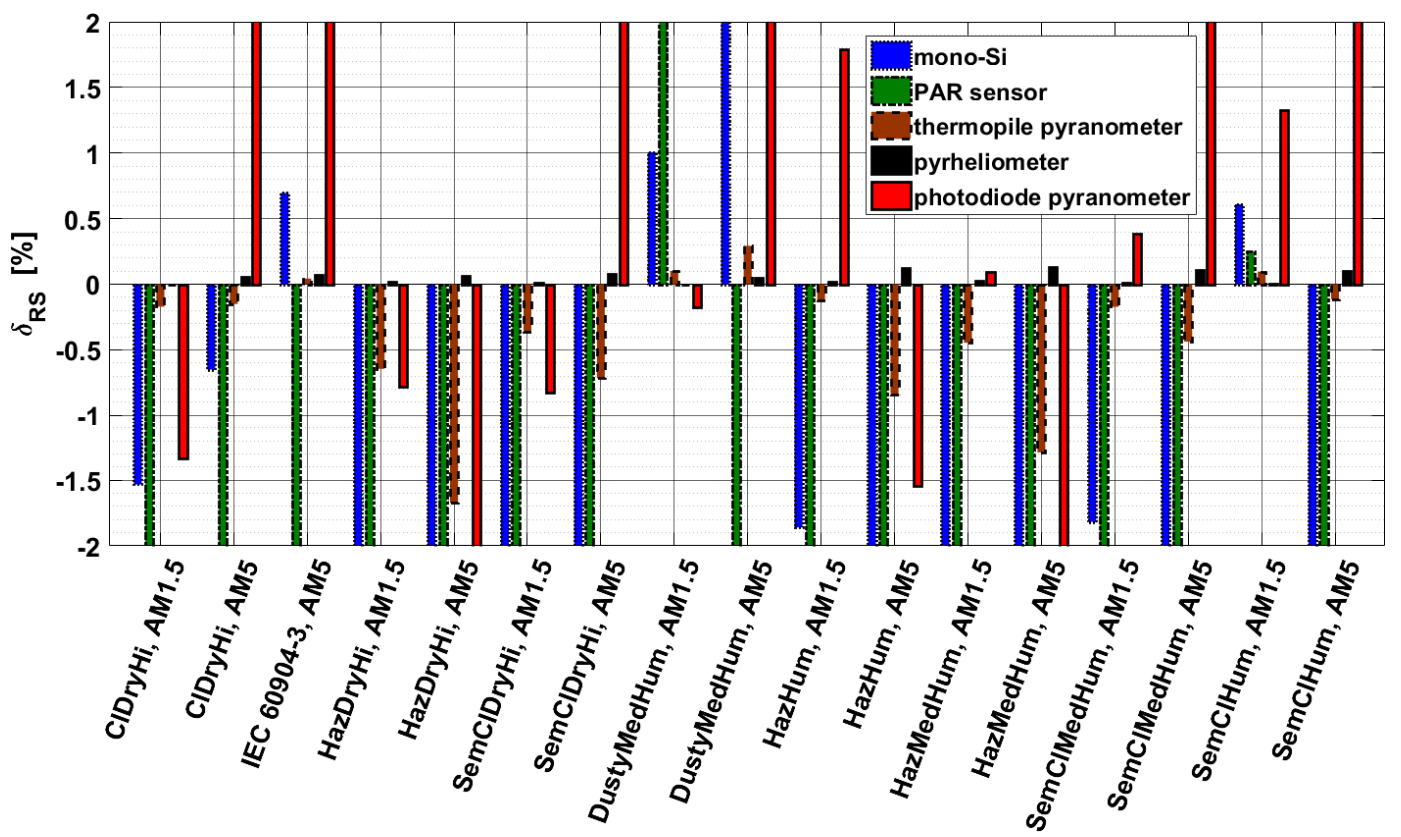

Fig. 7: Spectral errors for exemplary responsivities of radiometers and DNI. Same as Fig. 6 zoomed in. 
For DNI, the spectral errors are presented in Fig. 6. The results are also shown for hypothetical pyrheliometers with the spectral responsivity of a PAR sensor, a photodiode pyranometer, a mono-Si reference cell and a CMP11 like pyranometer as shown in Fig. 3. The corresponding clear sky spectral DNI errors are shown in Tab. 2. Huge errors of $-64 \%$ are visible for the PAR sensor and even for the photodiode pyranometer $10 \%$ error are found. This explains the absence of such DNI sensors on the market. Figure 7 shows the spectral errors for DNI after zooming in to $+/-2 \%$ spectral errors. The spectral errors of CHP1 like thermopile pyrheliometers are below $0.15 \%$ due to their quartz windows. A pyrheliometer using cheaper NBK7 entrance windows that are commonly used for thermopile pyranometers would result in spectral errors of up to $1.7 \%$. Also working with different less adequate Infrasil quartz glass types would result in higher clear sky spectral DNI errors of up to $0.7 \%$ (not shown). This further demonstrates the efficiency of the method to distinguish between radiometer classes in terms of the spectral error.

\section{Robustness of the method against deviations of the spectral responsivity}

An interesting question regarding this classification method is its robustness against variability of the spectral responsivity. The spectral responsivity of individual instruments of the same model might deviate from each other. Furthermore, spectral responsivity measurements for the common photodiode pyranometer LI-200 by NREL (Vignola et al., 2016) indicate that the manufacturer specification might not be accurate. This could also be the case for other manufacturer specifications.

Figure 8 shows the spectral responsivities for the LI-200 from the manufacturer specification and NREL's measurements (Vignola et al., 2016). Also the spectral responsivities of the similar, but newer LI-200R pyranometer and a mono-Si cell are shown. One can notice significant deviations, especially to the NREL measurement and to the reference cell. To test the effect on the classification, we calculated the clear sky spectral GHI errors for the four spectral responsivities (Fig. 9, left). Approximately $0.5 \%$ difference are seen between the LI-200's error depending on the used data set. More than $1 \%$ difference are found between the newer LI-200R and the Li-200 calculated using NREL data. However, the clear sky errors of all four devices are all within the limits of $5 \%$ and $1 \%$ which are established for the pyranometer classes $\mathrm{C}$ and B. Although in some cases the limits might be surpassed due to the deviations of spectral responsivity data, this quite extreme example shows a sufficient robustness of the classification scheme.

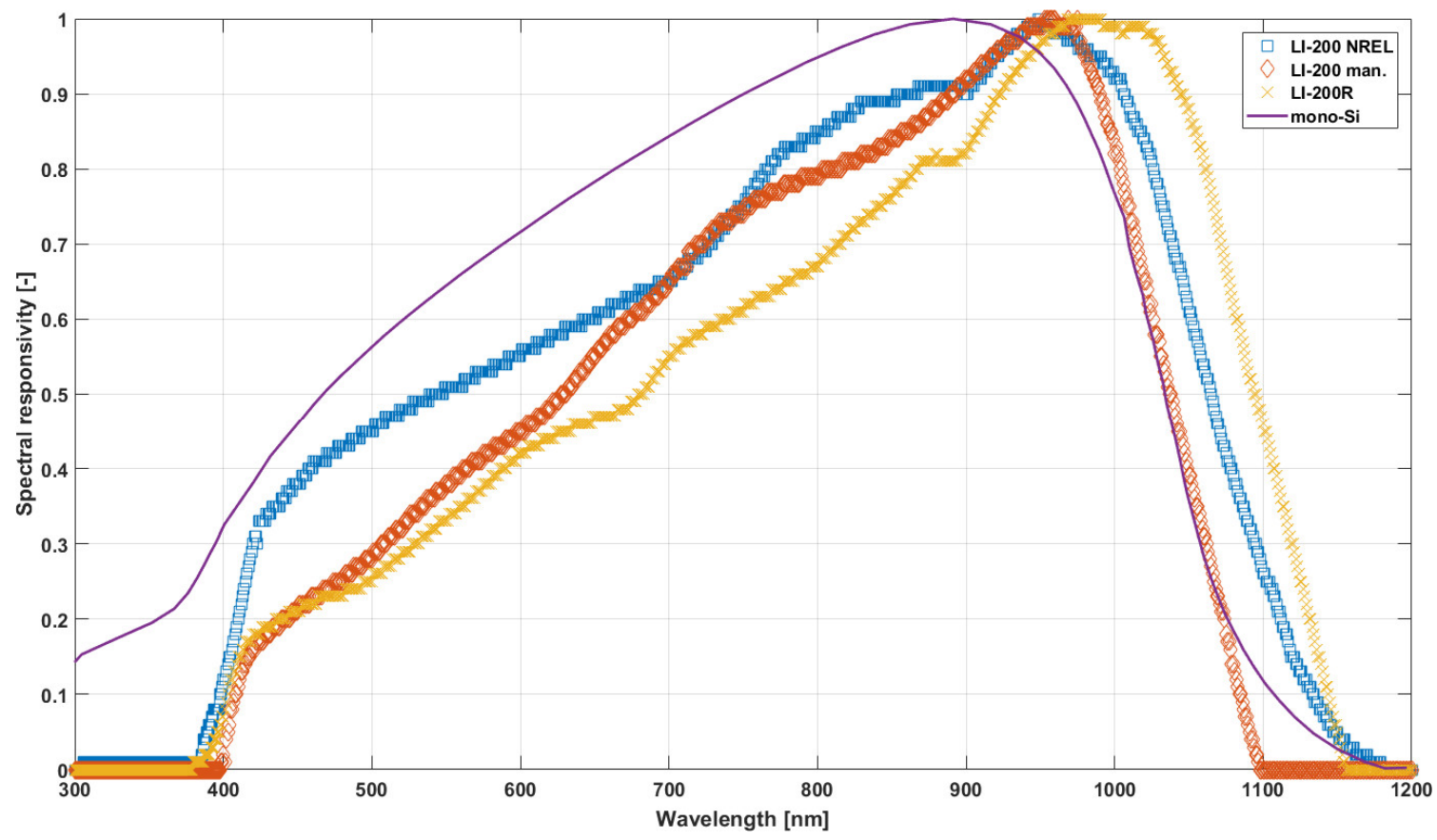

Fig. 8: Spectral response for mono-Si cell, the new LI-200R and the LI-200 pyranometer using 2 different data sources. 

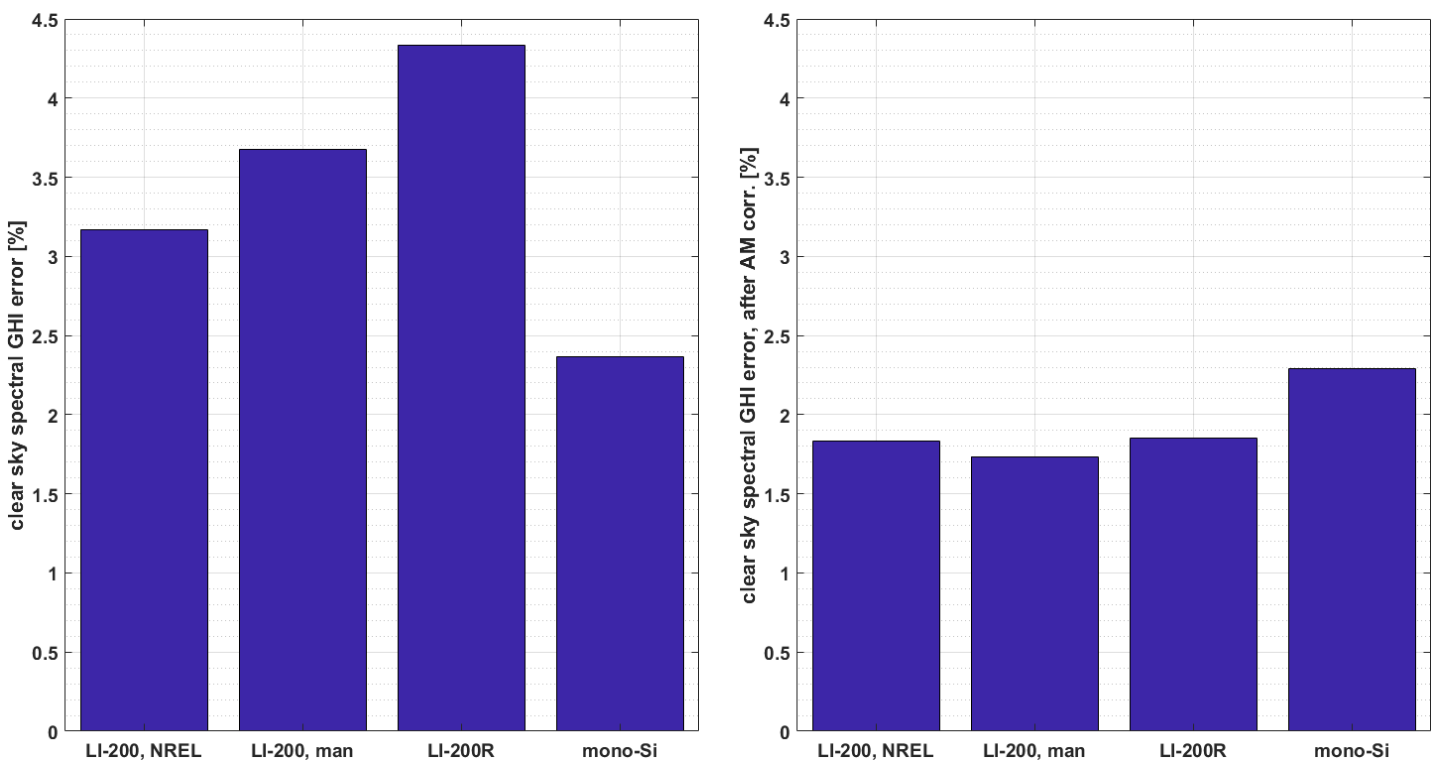

Fig. 9: Spectral errors for the LI-200 photodiode pyranometer calculated with spectral responsivity data from two different references, the newer LI-200R pyranometer and a mono-Si cell. Left: Without the application of any correction function. Right: After applying correction functions designed to minimize the clear sky spectral error as defined in ISO 9060:2018 based on AM.

\section{Clear sky spectral error for instruments using correction functions}

Another major change in the 2018 version of the ISO 9060 standard compared to previous version is that correction functions are allowed and that the corrected instrument signal can be used for the classification of the instrument. This also includes correction functions for the spectral errors. This correction must be available also for normal field operation, so that knowledge of the test spectra cannot be used. Corrections can be based for example on additional measurements, or current time, coordinates and altitude which allow the AM to be calculated. For example, $R_{\lambda}$ in eq. 1 could be multiplied by a factor obtained as a function $f$ of the AM. This leads to a correction of the uncorrected GHI $\left(G H I_{\text {raw }}\right)$ so that the corrected GHI is obtained as:

$$
G H I_{\text {corr }}=G H I_{\text {raw }} \cdot f(A M) .
$$

The possibility to include correction functions raises another important question: Can one design a simple correction function that allows a sensor to reach a higher accuracy class, although the correction might not work well in practice? We investigate the question for the four spectral responsivities from Fig. 8 designing the optimal corrections for each of the four devices that minimize the clear sky spectral error. We assume that only the $\mathrm{AM}$ is used as input parameter of the correction and that the correction uses eq. 2. In Fig. 4 it was clear that the spectral errors for AM 1.5 are much smaller than those for AM 5 and therefore we preliminarily set $f(A M=1.5)$ to 1 . Then we minimize the spectral errors for AM 5. For each device we use the vector $\vec{\delta}_{\mathrm{RS}}$ composed out of the $9 \delta_{\mathrm{RS}}$ for the $9 \mathrm{GHI}$ test spectra for AM 5 to determine the optimal correction according to

$$
f(A M=5)=\left[1+0.5 \cdot\left(\max \left(\vec{\delta}_{\mathrm{RS}}\right)+\min \left(\vec{\delta}_{\mathrm{RS}}\right)\right)\right]^{-1} .
$$

The thus obtained $f(A M=5)$ are shown in Tab. 3. The remaining spectral errors are calculated and the clear sky spectral GHI error is still determined by the AM 5 spectra for all four devices. Therefore, the preliminary setting of $f(A M=1.5)=1$ can be used for the optimal air mass based correction.

Tab. 3: Correction factor $f(A M=5)$ for the GHI correction of the four investigated devices.

\begin{tabular}{cc}
\hline Device & $\boldsymbol{f}(\boldsymbol{A M}=\mathbf{5})$ \\
\hline LI-200 NREL & 0.987 \\
LI-200 man. & 0.981 \\
LI-200R & 0.976 \\
mono-Si cell & 0.999
\end{tabular}


The clear sky spectral GHI error after this correction is shown in Fig. 9, right. Compared to the uncorrected clear sky spectral GHI errors from Fig. 9, left one can see a noticeable improvement for the photodiode pyranometers from the range of 3 to $4.5 \%$ to about $1.8 \%$ after the correction. This indicates the potential of such correction functions as also discussed in the literature (Forstinger et al., 2019, Geuder et al., 2016, Vignola et al., 2017). The mono-Si cell is only improved less from $2.4 \%$ to $2.3 \%$. This different behavior compared to the photodiode pyranometers can be explained by the different pattern of the spectral errors $\delta_{\mathrm{RS}}$ for AM 5 as seen in Fig. 4. For AM 5 the LI-200R always overestimates the GHI while the mono-Si cell shows overestimation and underestimation for different spectra with AM 5. Therefore, the AM based correction factor is close to 1 and cannot significantly reduce the clear sky spectral error of the mono-Si cell.

All four devices remain within the established limits of $1 \%$ and $5 \%$ for the clear sky spectral GHI error of class $\mathrm{B}$ and $\mathrm{C}$ pyranometers. This is important as a simple correction as in the example is not expected to improve the actual measurement accuracy significantly. Therefore, we expect that the accuracy class remains unchanged after the application of this simple correction. The significant remaining spectral errors show the strong influence of the atmospheric conditions on the spectra and the spectral errors. The same test has also been performed for the PAR sensor. Its clear sky spectral GHI error remains well above the $5 \%$ limit class $\mathrm{C}$ pyranometers, so that the classification remains unchanged, too. The tests for the AM correction indicate a sufficient robustness of the classification method even if correction functions are applied.

\section{Conclusion and discussion}

The method used in ISO 9060:2018 to derive the clear sky spectral irradiance error for the classification of pyrheliometers and pyranometers has been presented. The spectral errors for common radiometers and a monoSi reference cell were shown. The classification method using the clear sky spectral errors can easily separate different devices such as photodiode pyranometers and thermopile pyranometers in adequate classes. The spectral error of photodiode pyranometers is relevant as it lies in the order of magnitude of their directional errors and calibration errors. Furthermore, the classification method allows to exclude instruments such as PAR sensors from their classification as pyranometers. Also common photodiodes and reference cells are excluded from the application as pyrheliometers using the classification method.

The classification using the clear sky spectral error was tested for robustness against deviations in the used spectral selectivity data in order to check its reliability. This test was conducted with different spectral selectivity data for the LI 200 Silicon photodiode pyranometer, for the newer LI-200R photodiode pyranometer and a mono-Si cell. Despite of significant deviations of the spectral selectivities of the four devices all of them are in the same ISO 9060:2018 category of clear sky spectral errors. This indicates a sufficient robustness of the method against deviations of the spectral responsivity data.

As the new classification also allows the application of correction functions, the spectral errors were also derived for the mentioned four Silicon devices after applying a simple spectral correction. This correction was designed to minimize the clear sky spectral GHI error using the AM as input. The correction was able to reduce the clear sky spectral GHI errors significantly in the case of the photodiode pyranometers, but the resulting clear sky spectral GHI errors are still far from the limit specified for reaching a higher accuracy class. This is a first indication for a sufficient robustness of the classification method against unrealistic classifications due to the application of correction functions.

The classification using the clear sky spectral irradiance error is considered a significant advantage compared to the classification using the spectral selectivity as in the 1990 version of the ISO 9060 standard. The previous version of the 9060 standard completely excluded photodiode pyranometers due to their spectral selectivity. Furthermore, the specification of the spectral selectivity for an instrument was difficult to translate to a measurement error. A huge spectral selectivity can even be connected to no spectral error at all. This would be the case if the spectral responsivity of a radiometer is completely flat except of a strong deviation in a wavelength interval in which the spectral irradiance is zero (e.g. around $1380 \mathrm{~nm}$ ). The new clear sky spectral irradiance error at least gives an indication of typically occurring GHI and DNI errors due to the spectral variation under clear sky conditions. The method does not provide spectral errors for 1) cloudy situations, 2) extreme conditions not covered by the use cases, and 3) diffuse, reflected or tilted irradiance measurements. This is important for the understanding of measurement errors, but not necessary for the instrument 
classification.

There is also another classification scheme for radiometers published by WMO (WMO, 2014). This classification was close to the 1990 version of the ISO 9060, but for the spectral selectivity a decisive deviation was present. The wavelength range considered in the spectral selectivity definition by WMO is 300 to $3000 \mathrm{~nm}$ while it was 350 to $1500 \mathrm{~nm}$ in the 1990 version of ISO 9060. The WMO limits for the selectivity for the different classes were the same or even stricter as in the case of the highest pyranometer class. This leads to the unfortunate situation that to our knowledge no weather proof pyrheliometer fulfills the requirements of the WMO classes. Although the spectral errors of the tested pyrheliometer are small $\left(\delta_{\mathrm{RS}}<0.1 \%\right)$ the spectral selectivity according to the WMO definition is $2.5 \%$ for this pyrheliometer. This is much higher than the limit of $1 \%$ established for the lowest pyrheliometer class. Typical pyranometers of the highest ISO 9060 class are also excluded from the WMO classification. This holds for both the 1990 and the 2018 version of the ISO 9060 standard. For example, the responsivity of the class A thermopile pyranometer investigated in this work falls to about $12 \%$ at $3000 \mathrm{~nm}$ which leads to a very high spectral selectivity according to the WMO definition. We recommend that the WMO radiometer classification scheme is also adapted to the new 2018 version of the ISO 9060 .

The new classification method for the spectral irradiance errors was found to be a great improvement compared to previous methods and its results are considered as adequate. Future work related to the spectral errors after using sophisticated correction functions, spectral errors under cloudy conditions and for diffuse, reflected or tilted irradiance are of interest for solar energy.

\section{Acknowledgments}

We thank Dr. Chris Gueymard for providing the SMARTS software which allowed the calculation of the underlying spectra. We are thankful to the European commission, the Helmholtz Association and the German Federal Ministry for Economic Affairs and Energy for partly funding this work. We thank Kipp \& Zonen and Mr. Leo van Wely for providing us spectral absorptance data for the thermopile coating. We are grateful for the fruitful discussions with the members of ISO technical committee 180 , scientific committee 1 related to the spectral error.

This work was authored in part by the National Renewable Energy Laboratory, operated by Alliance for Sustainable Energy, LLC, for the U.S. Department of Energy (DOE) under Contract No. DE-AC3608GO28308. Funding provided by the U.S. Department of Energy Office of Energy Efficiency and Renewable Energy Solar Energy Technologies Office. The views expressed in the article do not necessarily represent the views of the DOE or the U.S. Government. The U.S. Government retains and the publisher, by accepting the article for publication, acknowledges that the U.S. Government retains a nonexclusive, paid-up, irrevocable, worldwide license to publish or reproduce the published form of this work, or allow others to do so, for U.S. Government purposes.

\section{References}

IEC 60904-3:2016, Photovoltaic devices — Part 3: Measurement principles for terrestrial photovoltaic (PV) solar devices with reference spectral irradiance data. International Standard.

ISO 9060:1990. 1990. Solar energy - Specification and classification of instruments for measuring hemispherical solar and direct solar radiation. International Standard.

ISO 9060:2018. 2018. Solar energy - Specification and classification of instruments for measuring hemispherical solar and direct solar radiation. International Standard.

Jessen, Wilko, Stefan Wilbert, Christian A. Gueymard, Jesús Polo, Zeqiang Bian, Anton Driesse, Aron Habte, Aitor Marzo, Peter R. Armstrong, Frank Vignola, and Lourdes Ramírez. 2018. "Proposal and evaluation of subordinate standard solar irradiance spectra for applications in solar energy systems." Solar Energy no. 168:3043. doi: https://doi.org/10.1016/j.solener.2018.03.043.

Forstinger, Anne, Stefan Wilbert, Anton Driesse, Natalie Hanrieder, Roman Affolter, Neeraj Goswami, Sharad Kumar, Norbert Geuder, Frank Vignola, Luis Zarzalejo, and Aron Habte. 2019. "Physically based correction of 
systematic errors of Rotating Shadowband Irradiometers." submitted to Meteorologische Zeitschrift.

Geuder, Norbert, Roman Affolter, Olaf Goebel, Basel Dahleh, Mohamed Al Khawaja, Stefan Wilbert, Benedikt Pape, and Benedikt Pulvermueller. 2016. "Validation of Direct Beam Irradiance Measurements From Rotating Shadowband Irradiometers in a Region With Different Atmospheric Conditions." Journal of Solar Energy Engineering no. 138 (5):051007-051007. doi: 10.1115/1.4034070.

Gueymard, C. 1995. SMARTS2, a simple model of the atmospheric radiative transfer of sunshine: algorithms and performance assessment. Rep. FSEC-PF-270-95 Florida Solar Energy Center, Cocoa, Fla.

Gueymard, Christian, 2001. Parameterized transmittance model for direct beam and circumsolar spectral irradiance. Solar Energy 71 (5):325-346.

Heraeus, 2019. Spectral Transmittance data for a $2 \mathrm{~mm}$ Infrasil 301 window. https://www.heraeus.com/en/hca/fused silica quartz knowledge base 1/t calc 1/transmission calculator hca. html?selection=reflection losses, infrasil 301_302\&chart=0\&rangeX $=120,5000 \&$ rangeY $=0,100$. Last accessed 23.7.2019.

Kipp\&Zonen. 2006. Instruction Manual for CMP Series Pyranometers and CMA Albedometers (version 0806).

Kipp\&Zonen. 2008. CHP1 Pyrheliometer Instruction Manual (version 0811).

Kipp\&Zonen. 2009. CH1 Pyrheliometer Instruction Manual (version 0901).

LICOR Biosciences, 2005. LICOR Terrestrial Radiation Sensors Instruction Manual. LICOR, Nebraska.

LICOR. 2015. Light Measurement. Report 980-15539 06/15. LICOR, Nebraska.

Vignola, Frank, Zachary Derocher, Josh Peterson, Laurent Vuilleumier, Christian Félix, Julian Gröbner, and Natalia Kouremeti. 2016. "Effects of changing spectral radiation distribution on the performance of photodiode pyranometers." Solar Energy no. 129:224-235. doi: http://dx.doi.org/10.1016/j.solener.2016.01.047.

Vignola, Frank, Josh Peterson, Stefan Wilbert, Philippe Blanc, Norbert Geuder, and Chris Kern. 2017. "New methodology for adjusting rotating shadowband irradiometer measurements." AIP Conference Proceedings no. 1850 (1):140021. doi: 10.1063/1.4984529.

Wilbert, Stefan, Stefan Kleindiek, Bijan Nouri, Norbert Geuder, Aron Habte, Marko Schwandt, and Frank Vignola. 2016. "Uncertainty of rotating shadowband irradiometers and Si-pyranometers including the spectral irradiance error." AIP Conference Proceedings no. $1734 \quad$ (1):150009. doi: doi:http://dx.doi.org/10.1063/1.4949241.

Wilbert, Stefan, Wilko Jessen, Christian Gueymard, Jesús Polo, Zeqiang Bian, Anton Driesse, Aron Habte, Aitor Marzo, Peter Armstrong, Frank Vignola, and Lourdes Ramírez. 2017. "Proposal and Evaluation of Subordinate Standard Solar Irradiance Spectra with a Focus on Air Mass Effects." Solar World Congress. doi: doi:10.18086/swc.2017.21.06

Winter, S., Friedrich, D., and Sperling, A., Effects of the new standard IEC 60904-3:2008 on the calibration results of common solar cell types. 24th European Photovoltaic Solar Energy Conference, 21-25 September 2009, Hamburg, Germany. WMO. 2014. Guide to Meteorological Instruments and Methods of Observation. WMO-No. 8, 2014 Update. Eighth ed.. 